\title{
Factors Influencing Infant and Adolescent Vaccine Uptake in Flanders, Belgium
}

H. Theeten, E. Lefevere, C. Vandermeulen, P. Van Damme, and N. Hens

\begin{abstract}
This chapter focuses on the determinants of a number of immunization 4 programme outcomes in Flanders (Belgium), such as vaccine initiation and uptake, 5 completion of the vaccination schedule and compliance to official validity criteria. 6 These were assessed in both infant and adolescent age groups. Three main groups 7 of potential influencing factors are looked at: (1) individual background variables; 8 (2) family level variables; (3) external factors such as the governmental vaccination 9 programme and other initiatives to promote vaccination. Data on parental willing- 10 ness to pay for and willingness to accept multiple concomitant injections and their 11 determinants are discussed as well. Exploring relationships between vaccination 12 programme outcomes and influencing factors can give important information to 13 optimize vaccination programme performance.

H. Theeten $(\bowtie) \cdot \mathrm{P}$. Van Damme

Centre for the Evaluation of Vaccinations, Vaccine and Infectious Disease Institute, University of Antwerp, Universiteitsplein 1, 2610 Wilrijk, Belgium

e-mail: heidi.theeten@ua.ac.be; pierre.vandamme@ua.ac.be

E. Lefevere,

Herman Deleeck Centre for Social Policy, University of Antwerp, St Jacobstraat 2,

2000 Antwerp, Belgium

e-mail: eva.lefevere@ua.ac.be

C. Vandermeulen

Department of Youth Health Care, KU Leuven, Kapucijnenvoer 35, 3000 Leuven, Belgium

e-mail: corinne.vandermeulen@uzleuven.be

N. Hens

Center for Statistics, Hasselt University, Agoralaan 1, 3590 Diepenbeek, Belgium

Centre for Health Economic Research and Modeling Infectious Diseases, University

of Antwerp, Universiteitsplein 1, 2610 Wilrijk, Belgium

e-mail:niel.hens@uhasselt.be

P. Manfredi and A. d'Onofrio (eds.), Modeling the Interplay Between Human Behavior 


\section{Introduction}

During the previous century, highly effective vaccines have been developed and 16 vaccination programmes have been implemented. Together, these have allowed for 17 primary prevention of infectious diseases that once disabled or killed large numbers 18 of adults and children, such as measles, polio and diphtheria. However, continued 19 and extensive surveillance of diseases, vaccines and vaccination programmes is 20 necessary since any of these diseases can be reintroduced, as has happened in the 21 past. Such reintroduction of diseases can be induced by, among others, vaccine 22 failure or failure to vaccinate for reasons such as programme performance regression 23 or changes in people's attitudes and perceptions towards vaccinations. Accurate 24 information on vaccine uptake, disease susceptibility in the target groups, disease 25 epidemiology and changes in people's attitudes and preferences towards vaccines 26 and vaccination programmes is highly needed to evaluate the performance of 27 recommended vaccination programmes. This information can also be used to guide 28 decisions on adaptation of the existing programmes or on the introduction of new 29 vaccines.

This chapter summarizes findings from studies on infant and adolescent vac- 31 cination in Flanders, the northern region of Belgium, representing about $2 / 3$ of 32 the Belgian population. The studies explored various indicators of vaccine uptake 33 (vaccination initiation, vaccination completion), compliance with the recommended 34 validity criteria and attitudes and preferences towards vaccinations. In order to 35 understand the setting of these studies some background information on the 36 organization of the vaccination programme in Belgium seems appropriate. $\quad 37$

Belgium is governed both by a national and sub-national (regional) governments. 38 Vaccination policy is a shared responsibility of the national and the regional 39 Ministries of Health. A national schedule of recommended vaccines is provided 40 and regularly updated by the national Superior Health Council (SHC). The regional 41 authorities are responsible for the organization and promotion of the immunization 42 programmes in their respective regions. The way the vaccination programme is 43 organized in each region (supply of vaccines via the public and the private health 44 setting vs via the private health setting only) as well as the price of the vaccine 45 (free of charge, partially or not reimbursed) are jointly decided by the national 46 and the regional governments. With regard to the organization of the vaccination 47 programme, for most vaccines recommended by the SHC, parents can choose to 48 have their child vaccinated in a public or in a private health care setting. In the 49 case of infant vaccinations, the public health setting consists of well-baby clinics. 50 These clinics systematically offer vaccines to all children between 0 and 3 years 51 of age through regular preventive consultations. Most of these vaccines are free of 52 charge, while for some of them an out-of-pocket cost is required. In the case of 53 school-aged children, the public health setting consists of free of charge preventive 54 consultations by school health services. Throughout the school career of a child, the 55 vaccination status is checked at regular points in time and recommended vaccines 56 are offered systematically to children at specific ages. All vaccines offered by 57 these school health services are currently (situation April 2012) free of charge. 58 
The private health care setting for both infant and school-aged children consists 59 of general practitioners and paediatricians, who can order and administer free of 60 charge vaccines as well. Nevertheless, they charge a fee for the consultation. 61

In practice, many vaccines, once the SHC recommends them, are first available 62 only via the private health care setting during some time, prior to an agreement 63 on financing between the national and the regional governments. This was the 64 case for, e.g., the hepatitis B vaccine, the Haemophilus influenzae type b vaccine, 65 pneumococcal vaccines and human papilloma virus (HPV) vaccines. If no final 66 agreement is reached between the national and the regional government on a 67 specific vaccine, this vaccine can still be obtained via the private health setting, 68 but the initiative for vaccination lies entirely with the parents or with the physicians, 69 and there is no systematic offer of these vaccines to the eligible children. Partial 70 reimbursement is in some cases provided by the national government or by private, 71 non-profit sickness funds.

\section{Studies}

The results described in this section can be divided into two subsections. In the 74 first subsection, indicators of vaccine uptake and its determinants are described. 75 In the second subsection, we look at other indicators relevant for the surveillance and 76 monitoring of the vaccination programme, namely parental attitudes with regard to 77 the administration of concomitant vaccines and willingness to pay to avoid an extra 78 injection. Table 1 gives an overview of the different studies that are summarized 79 below.

\subsection{Determinants of Vaccine Uptake}

We first describe the indicators of vaccine uptake and its determinants for various 82 vaccines supplied via both the public and the private setting, and subsequently 83 indicators for one specific vaccine (HPV vaccine) during the period it was only 84 supplied via the private health care setting (partly reimbursed). For all vaccines we 85 identified low-uptake risk groups. Identification of low-uptake risk groups allows 86 for targeted strategies that can enhance the uptake of vaccines, overall or in certain 87 risk groups.

\subsubsection{Vaccines Offered Both via the Public and the Private Health Care Setting}

A first series of studies [1, 4, 7, 8] investigated indicators of vaccine uptake for 91 vaccines offered free of charge to infants and school children both via the public and 92 the private health care setting. Information was obtained through two immunization 93 


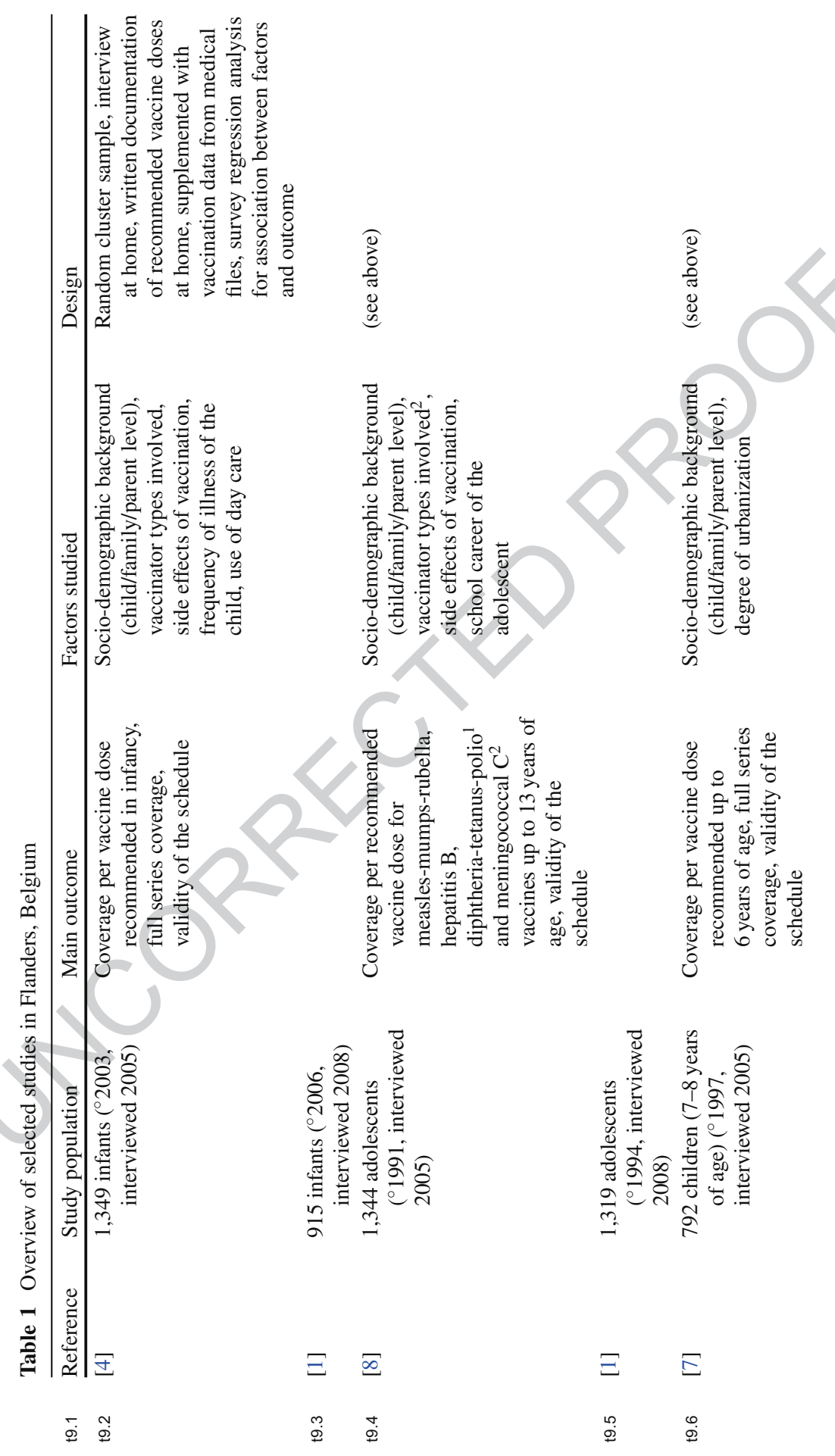




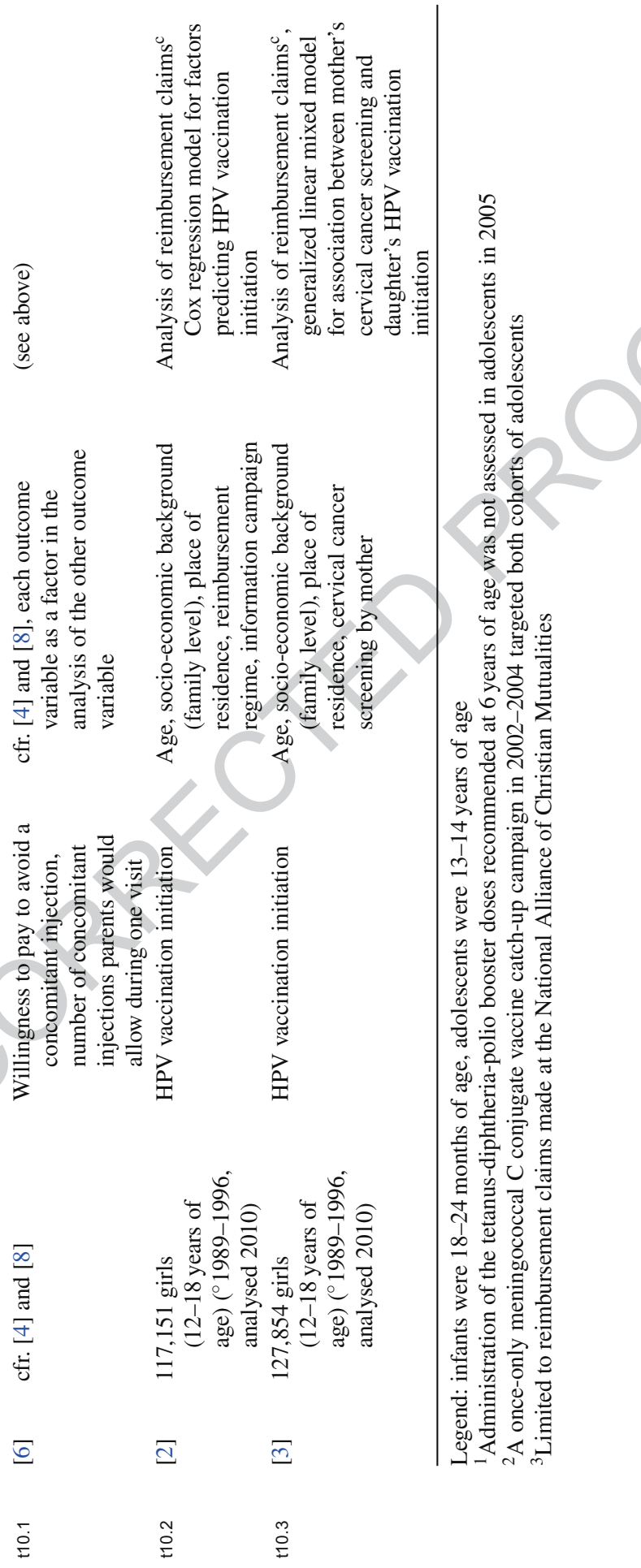


coverage surveys performed in 2005 and 2008, both ordered by the Flemish Ministry 94 of Health. Their principal aim was to assess coverage of the following infant 95 and adolescent vaccines: poliomyelitis (polio), diphtheria-tetanus-pertussis (DTP), 96 H. influenzae type b (Hib), hepatitis B (HBV), measles-mumps-rubella (MMR) 97 and meningococcal $\mathrm{C}(\mathrm{MenC})$ vaccines. The survey comprised samples of 18-24- 98 month-old infants ( ${ }^{\circ} 2003$ and $\left.{ }^{\circ} 2006\right)$, 7-8-year-old school-aged children $\left({ }^{\circ} 1997\right) 99$ and 13-14-year-old adolescents $\left({ }^{\circ} 1991\right.$ and $\left.{ }^{\circ} 1994\right)$ (Table 1$)$. Two-step random 100 cluster samples were selected as recommended by the Expanded Programme on 101 Immunization (EPI) of the World Health Organization. Families were interviewed 102 at home. The obtained vaccination data were updated from medical files of private 103 physicians or public health services (if necessary and if possible), as well as from 104 the child's individual record in Vaccinnet, Flanders' online vaccine ordering and 105 registration system. The main results with regard to vaccine uptake are summarized 106 in Table 2.

Vaccine coverage in Flanders was found to be higher at infant age (where it 108 surpassed $90 \%$ for all assessed vaccines) than at later age (where the coverage 109 of most recommended vaccines was below 90\%). Note that non-availability of 110 vaccination documents at home was also more frequent at later age and can thus 111 have biased the findings. First dose coverage (limited to multi-dose vaccines), an 112 indicator of vaccination initiation, ranged from 96.9 to $99.0 \%$ in infants and from 113 80.6 to $83.3 \%$ in adolescents in 2005. In 2008, vaccination initiation levels of 114 close to $100 \%$ and between 86.4 and $92.5 \%$ were noted in infants and adolescents, 115 respectively. Full series coverage (vaccination completion) per vaccine in infants 116 ranged from 92.2 to $94.1 \%$ in 2005 and from 95.1 to $96.6 \%$ in 2008. In adolescents, 117 full series were assessed for HBV and MMR vaccines only. Full series coverage 118 for MMR in this age group rose from 74.6 in 2005 to $83.5 \%$ in 2008; for 119 HBV a rise from 75.7 to $89.3 \%$ was noted. Age-appropriate vaccination rate (full 120 series of all recommended vaccines) in infants was stable at 89.5 and $89.6 \%$ in 121 2005 and 2008, respectively, whereas in adolescents it increased from 58.1 to 122 $72.8 \%$. Excluding invalid doses (as based on official criteria for minimal age at 123 administration and minimal interval between doses) resulted in a reduction of full 124 series coverage only in infants. Valid series coverage per vaccine in that age group 125 ranged from 85.6 to $90.1 \%$ in 2005 and from 88.4 to $93.4 \%$ in 2008. Several 126 predictors of the vaccination coverage per vaccine and per dose in each surveyed 127 age cohort were studied, using parametric and non-parametric methods (Table 2). 128 The most important predictor of lower vaccination coverage in infants was the main 129 vaccinator, with children vaccinated in the private health care setting having a higher 130 risk of undervaccination than children vaccinated in the public health care setting. 131 In adolescents an atypical school career was a consistent risk factor, but several 132 socio-economic factors were found to be significant as well, with, e.g., children from 133 families with a lower family income or children whose parents or grandparents were 134 born outside the EU having a higher risk of undervaccination. For a lot of factors 135 the association with coverage of specific vaccines was significant in one birth cohort 136 and borderline or non-significant in another, but in general similar trends could be 137 seen. 


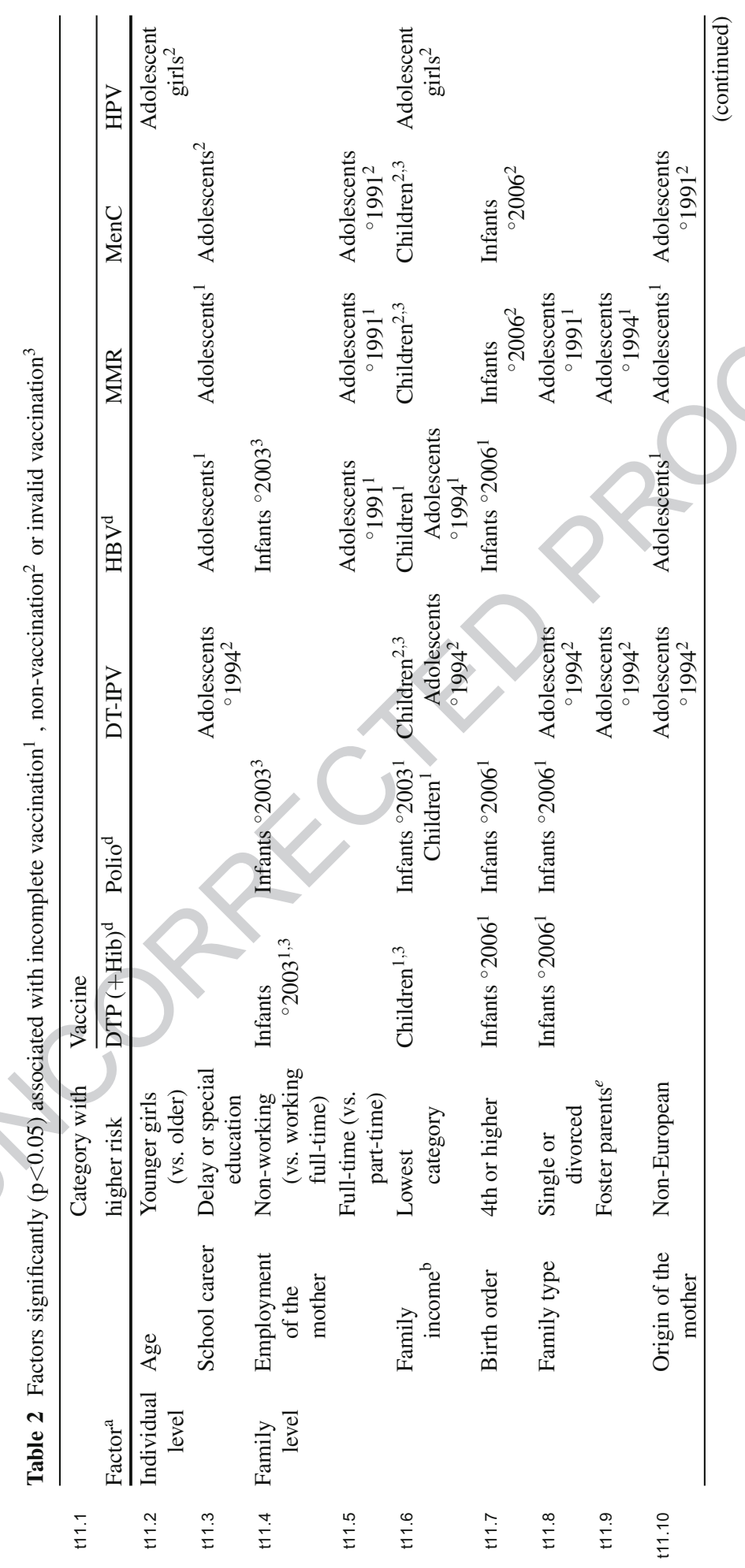




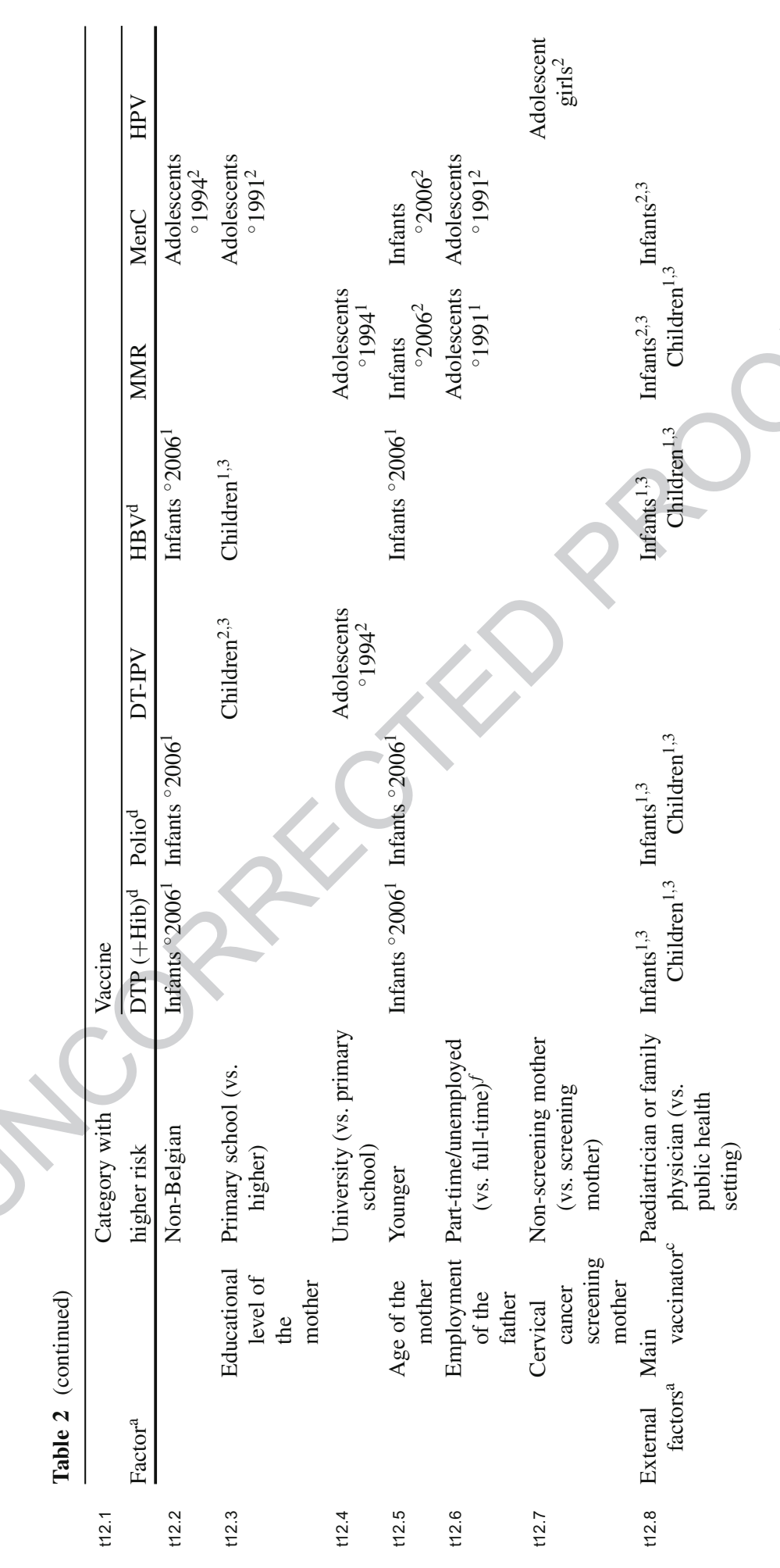




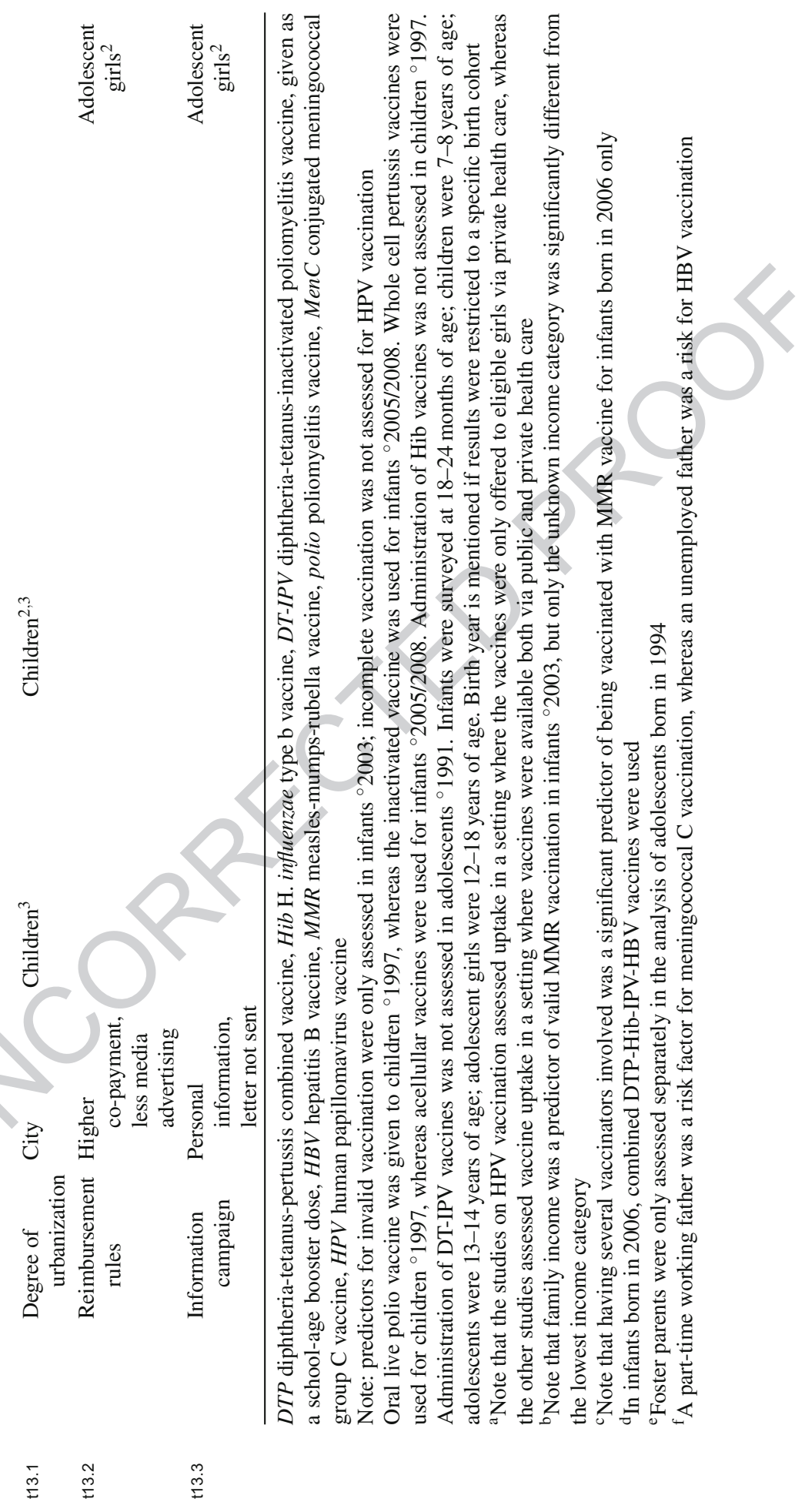




\subsubsection{Vaccines Offered Only via a Private Health Care Setting}

The second series of studies [5,6] investigated predictors of vaccine uptake for one 140 specific vaccine, namely the HPV vaccine, in the period it was recommended to 141 adolescent girls by the SHC but offered only via the private health care setting with 142 partial reimbursement (2007-2009). Information was obtained through analyses 143 of HPV vaccine reimbursement claims of the National Alliance of Christian 144 Mutualities (NACM), the largest sickness fund in Flanders. All female members 145 aged 12-18 ( ${ }^{\circ} 1989-1996 ; \mathrm{N}=117151$ in [2], $\mathrm{N}=127854$ in [3]) and living in 146 Flanders were selected from the membership files of the NACM. Initiation of HPV 147 vaccination between January 2007 and June 2009 varied between 20 and $80 \% 148$ depending on the year of birth (age) of the girls. These differences in vaccination 149 coverage were mainly due to two factors. First, there were differences in the 150 reimbursement rules (during certain periods of time and for certain birth cohorts 151 the out-of-pocket cost for the vaccines was much lower, and eligibility rules were 152 more advertised in the media). Second, the vaccine was partly reimbursed up until 153 the age of 15 or later 18 years, so for the youngest girls in the study (born in 1995 and 154 1996) there was still a lot of time left after June 2009 to start vaccination. Besides 155 these two main factors a higher probability of initiation of HPV vaccination was 156 found for girls coming from families with higher incomes and for girls who were 157 personally informed about their eligibility for reimbursement. Furthermore, the 158 probability of initiation of HPV vaccination was found to be positively associated 159 with cervical cancer screening of the mother in the years prior to the launch of HPV 160 vaccines (factors affecting the probability of vaccination initiation are summarized 161 in Table 2).

\subsection{Parental Attitudes and Willingness to Pay}

The main results with regard to parental attitudes are summarized in Table 3. 164

In the vaccination coverage study of 2005 additional questions were added to the 165 questionnaires of both infants and adolescents to obtain information on parental 166 attitudes and preferences with respect to multiple vaccine injections [5, 7]. The 167 results were analysed separately for parents of infants and parents of adolescents. 168 Willingness to pay (amount in euro) to avoid a concomitant injection and the 169 maximum number of concomitant injections parents would allow during the same 170 visit were used as a proxy of parental acceptance of concomitant injections. Parents 171 of young children as well as those of adolescents gave preference to a maximum 172 of two separate vaccine injections to be given at the same immunization visit. 173 Parents also shared common attitudes on the amount of money they would pay to 174 avoid concomitant injections. A significant proportion of parents of both infants 175 and adolescents, $41.0 \%$ and $38.8 \%$, respectively, were not willing to pay anything, 176 whereas in both age groups the remaining parents mentioned a median amount of 177 20 euro to avoid a concomitant injection. However, extensive analysis using several 178 regression methods to identify predictors of the attributed value and the allowed 179 
Table 3 Predictors of different proxies for parental acceptance of concomitant vaccine injections (not vaccine specific). Being willing to pay to avoid a concomitant injection (WTP); amount (in euro) caregivers are willing to pay (amount WTP); number of concomitant injections caregivers would allow (number allowed); as assessed in parents of infants and adolescents in 2005

\begin{tabular}{|c|c|c|c|c|c|}
\hline Predictor & $\begin{array}{l}\text { Category with lower } \\
\text { outcome }\end{array}$ & WTP Yes & $\begin{array}{l}\text { Amount } \\
\text { WTP }\end{array}$ & $\begin{array}{l}\text { Number } \\
\text { allowed }\end{array}$ & $\mathrm{t} 14.1$ \\
\hline $\begin{array}{l}\text { Educational level of the } \\
\text { mother }\end{array}$ & $\begin{array}{l}\text { Lowest (vs. secondary } \\
\text { school) }\end{array}$ & Infants & & & $\mathrm{t} 14.2$ \\
\hline \multirow[t]{2}{*}{ Employment of the father } & $\begin{array}{l}\text { Part-time or freelance } \\
\quad \text { (vs. full-time) }\end{array}$ & Infants & & & $\mathrm{t} 14.3$ \\
\hline & $\begin{array}{l}\text { Not working } \\
\quad \text { (vs. full-time) }\end{array}$ & Adolescents & & & $\mathrm{t} 14.4$ \\
\hline Number of siblings & Lower number & & & Infants & $\mathrm{t} 14.5$ \\
\hline \multirow[t]{2}{*}{ Origin of the mother } & $\begin{array}{l}\text { Belgian vs. other } \\
\text { European country }\end{array}$ & & $c$ & & $\mathrm{t} 14.6$ \\
\hline & $\begin{array}{l}\text { Non-European vs. } \\
\text { Belgian }\end{array}$ & & & & $\mathrm{t} 14.7$ \\
\hline Main vaccinator & $\begin{array}{l}\text { Well-baby clinic } \\
\text { (vs. paediatrician) }\end{array}$ & & Iant & & $\mathrm{t} 14.8$ \\
\hline $\begin{array}{l}\text { Number of concomitant } \\
\text { injections parents } \\
\text { would allow }\end{array}$ & Lower number & & & & $\mathrm{t} 14.9$ \\
\hline WTP & Being willing to $\mathrm{p}$ & & & Infants & $\mathrm{t} 14.10$ \\
\hline $\begin{array}{l}\text { Respondent's relation to } \\
\text { the child }\end{array}$ & $\begin{array}{l}\text { Mother vs } \\
\quad \text { grandparent }^{1}\end{array}$ & & & Infants $^{2}$ & $\mathrm{t} 14.11$ \\
\hline Child's vaccination status & $\begin{array}{l}\text { Complete } \\
\text { Incomplete }\end{array}$ & & & $\begin{array}{l}\text { Infants }^{2} \\
\text { Adolescents }^{3}\end{array}$ & $\begin{array}{l}\mathrm{t} 14.12 \\
\mathrm{t} 14.13\end{array}$ \\
\hline
\end{tabular}

Note: Only factors and categories with significant odds ratios were plotted. Family income was a significant predictor of WTP in infants, but only if the unknown income category was compared to the categories with known income

${ }^{1}$ Grandparents accounted for less than $2 \%$ of the respondents

${ }^{2}$ Comparing respondents who would allow an unlimited number of injections to those who would allow a limited number

${ }^{3}$ Comparing respondents who would allow more than two concomitant injections to respondents who would allow not more than two

number of concomitant injections (Table 3) explained only a small part of the 180 variability in the answering behaviour and yielded some conflicting information; 181 this suggests that the proxies we used are only rough indicators of parental attitudes 182 on concomitant vaccines.

\section{Discussion}

In this chapter we summarized studies on predictors of infants' and adoles- 185 cents' vaccine uptake and attitudes of parents towards vaccination in Flanders 186 (Belgium). First, individual level characteristics, such as age and school career 187 
were found to significantly affect vaccination coverage. Second, various family 188 level characteristics such as family income, parental educational level or screening 189 behaviour by the mother, were also significantly associated with vaccine uptake. 190 A final set of predictors of vaccine uptake consisted of external factors such as 191 main vaccinator, information campaigns or the reimbursement rules. Exploring 192 relationships between vaccine uptake and these predictors can help to identify 193 subgroups with higher risk of undervaccination who merit special attention. It can 194 also be used to monitor existing vaccination programmes and to guide decisions on 195 changes in these vaccination programmes. Information on parental attitudes towards 196 different aspects of vaccination and vaccination programmes can further optimize 197 these decisions. The results of the presented analyses suggest, in general, a need to 198 monitor and support vaccinating activities of private vaccinators (paediatricians and 199 family physicians) and to develop specific strategies for families in an unfavourable 200 socio-economic situation, as well as for children in special education programmes. 201 Interventions to increase vaccine uptake in infants should address the importance 202 both of timely administration and of completion of the schedule, since similar 203 risk factors were found for invalid and for incomplete vaccination. Apart from the 204 socio-economical and individual predictors of vaccine uptake, documentation of 205 vaccination is a major hurdle in the assessment of vaccination coverage, especially 206 in older age groups, when vaccination is less an issue and is often scattered over 207 different vaccine providers. A cornerstone for good documentation and vaccination 208 practices throughout life is a centralized registration database, which is easily 209 accessible for all vaccine providers and which takes into account safety and privacy 210 issues.

Regular reassessment of vaccine coverage in different settings would provide the 212 opportunity to detect and interpret trends over time. Targeted research (e.g. using 213 a qualitative design) in known subgroups of undervaccinated children could add 214 information on more specific hurdles for vaccination, out of which tailored strategies 215 could be inferred. Ideally data on vaccination coverage should be complemented 216 by studies designed to estimate the serological level of immunity in the target 217 population, which is the indicator we are ultimately interested in when evaluating 218 the performance of vaccination programmes. To have a better insight in parents' 219 preferences regarding concomitant vaccine injections, sensitive quantifications 220 using a more appropriate design (e.g. discrete choice experiments) would confer 221 important additional insights.

Acknowledgements We thank all the families who participated in the vaccine coverage studies, 223 the physicians who supplied information and all other collaborators. The vaccine coverage studies 224 were funded by the Flemish government. For the studies on HPV vaccination initiation we thank 225 the NACM for its fruitful cooperation. Both studies on uptake of the HPV vaccine were financed 226 by the Research Foundation Flanders (FWO). 


\section{References}

1. Hoppenbrouwers, K., Vandermeulen, C., Roelants, M., Boonen, M., Van Damme, 229 P., Theeten, H., Depoorter, A.: Studie van de vaccinatiegraad bij jonge kinderen en adolescenten 230 in Vlaanderen in 2008. Technical report, 2009. URL http://www.zorg-en-gezondheid.be/ 231 cijfersinfectieziekten_en_vaccinaties.aspx 232

2. Lefevere, E., Hens, N., De Smet, F., Van Damme, P.: BMC Public Health 11, 470 (2011). doi: 233 10.1186/1471-2458-11-470. URL http://dx.doi.org/10.1186/1471-2458-11-470 234

3. Lefevere, E., Hens, N., Theeten, H., Van den Bosch, K., Beutels, P., De Smet, F., Van Damme, 235 P.: Vaccine 29, 8390-8396 (2011). doi: 10.1016/j.vaccine.2011.08.039. URL http://dx.doi.org/ 236 10.1016/j.vaccine.2011.08.039

4. Theeten, H., Hens, N., Vandermeulen, C., Depoorter, A.-M., Roelants, M., Aerts, 238 M., Hoppenbrouwers, K., Damme, P.V.: Vaccine 25, 4940-4948 (2007). doi: 239 10.1016/j.vaccine.2007.03.032. URL http://dx.doi.org/10.1016/j.vaccine.2007.03.032 240

5. Theeten, H., Hens, N., Aerts, N., Vandermeulen, C., Roelants, M., Hoppenbrouwers, 241 K., Damme, P.V., Beutels, P.: Pediatr. Infect. Dis. J. 28, 61-63 (2009). doi: 242 10.1097/INF.0b013e318184eea3. URL http://dx.doi.org/10.1097/INF.0b013e318184eea3 243

6. Theeten, H., Hens, N., Aerts, M., Vandermeulen, C., Roelants, M., Hoppen- 244 brouwers, K., Damme, P.V., Beutels, P.: Vaccine 27, 1964-1969 (2009). doi: 245 10.1016/j.vaccine.2009.01.096. URL http://dx.doi.org/10.1016/j.vaccine.2009.01.096 246

7. Theeten, H., Vandermeulen, C., Roelants, M., Hoppenbrouwers, K., Depoorter, A.-M., Van 247 Damme, P.: Acta Paediatr. 98, 1307-1312 (2009). doi: 10.1111/j.1651-2227.2009.01331.x. 248 URL http://dx.doi.org/10.1111/j.1651-2227.2009.01331.x 249

8. Vandermeulen, C., Roelants, M., Theeten, H., Depoorter, A.-M., Van Damme, P., 250 Hoppenbrouwers, K.: Pediatrics 121, e428-e434 (2008). doi: 10.1542/peds.2007-1415. 251 URL http://dx.doi.org/10.1542/peds.2007-1415 


\section{Part II \\ Modeling Behaviour Change in Response ${ }_{2}$ to Epidemic Threats ${ }_{3}$}




\title{
Modeling the Impact of Behavior Changes on the Spread of Pandemic Influenza
}

Sara Y. Del Valle, Susan M. Mniszewski, and James M. Hyman

\begin{abstract}
We use mathematical models to assess the impact of behavioral changes 4 in response to an emerging epidemic. Evaluating the quantitative and qualitative 5 impact of public health interventions on the spread of infectious diseases is a crucial 6 public health objective. The recent avian influenza (H5N1) outbreaks and the 20097 H1N1 pandemic have raised significant global concerns about the emergence of a 8 deadly influenza virus causing a pandemic of catastrophic proportions. Mitigation 9 strategies based on behavior changes are some of the only options available in the 10 early stages of an emerging epidemic when vaccines are unlikely to be available 11 and there are only limited stockpiles of antiviral medications. Mathematical models 12 that capture these behavior changes can quantify the relative impact of different 13 mitigation strategies, such as closing schools, in slowing the spread of an infectious 14 disease. Including behavior changes in mathematical models increases complexity 15 and is often left out of the analysis. We present a simple differential equation 16 model which allows for people changing their behavior to decrease their probability 17 of infection. We also describe a large-scale agent-based model that can be used 18 to analyze the impact of isolation scenarios such as school closures and fear- 19 based home isolation during a pandemic. The agent-based model captures realistic 20 individual-level mixing patterns and coordinated reactive changes in human behav- 21 ior in order to better predict the transmission dynamics of an epidemic. Both models 22 confirm that changes in behavior can be effective in reducing the spread of disease. 23 For example, our model predicts that if school closures are implemented for the
\end{abstract}

S.Y. Del Valle $(\bowtie) \bullet$ S.M. Mniszewski

Los Alamos National Laboratory, West Jemez Road, Los Alamos, NM 87545, USA

e-mail: sdelvall@lanl.gov;smm@lanl.gov

J.M. Hyman

Tulane University, St Charles Avenue, New Orleans, LA, USA

e-mail: mhyman@tulane.edu 
duration of the pandemic, the clinical attack rate could be reduced by more than 24 $50 \%$. We also verify that when interventions are stopped too soon, a second wave of 25 infection can occur.

\section{Introduction}

Pandemics are global epidemics and are often associated with a high morbidity and 28 mortality burden. There have been three pandemic influenza outbreaks in the 20th 29 history: the Spanish flu (1918-19), the Asian flu (1957-58), and the Hong Kong 30 flu (1968-69) [32]. The 1918-1919 influenza pandemic (known as the Spanish flu) 31 was the most devastating in recent history, where at least 20 million died [30]. In the 32 United States, about 675,000 lives were lost to the Spanish flu with an estimated 33 mean case fatality rate of $2 \%$ [52]. This case fatality rate is an order of magnitude 34 larger than the case fatality rates observed in seasonal flu epidemics in normal 35 years. Recurrent outbreaks of H5N1 around the world and the most recent pandemic 36 (H1N1) 2009 suggest that a deadly pandemic is eminent.

Nearly half of the world's population resides in urban areas [50]. Air travel 38 connects these urban centers in a global network where a new influenza strain can 39 spread around the world in a few weeks, as recently experienced with pandemic 40 (H1N1) 2009. In addition, influenza's short incubation period and the lack of a 41 universal vaccine can increase the spread of influenza, posing a significant global 42 challenge to public health officials. Mathematical models can help in meeting this 43 challenge, if the model includes the most significant properties of the transmission 44 dynamics. In particular, the model most include how people change their behavior 45 in response to an epidemic threat.

Evidence suggests that in the presence of a deadly disease and lack of 47 pharmaceutical interventions, people will change their behavior to avoid infection 48 $[15,19,42]$. Recent studies have evaluated the impact that non-pharmaceutical 49 interventions, such as school closures, social distancing, and travel restrictions, 50 could have on the spread of the next influenza pandemic [13, 14, 21, 24]. However, 51 none of these studies have incorporated intentional changes in individual behavior, 52 such as avoiding gatherings, increasing hygiene, or staying home. Furthermore, 53 these studies have assumed that these non-pharmaceutical interventions would 54 remain in effect for the duration of the pandemic. Typically, people resume their 55 normal behaviors due to lack of resources or as the perceived risk declines [27]. 56 Recent studies on the impact of basic public health measures implemented during 57 the 1918 pandemic $[6,27]$ indicate that non-pharmaceutical interventions did not 58 last for the duration of the pandemic.

Mathematical models for the spread of infectious diseases have been extensively 60 used to gain insights into the transmission dynamics of infectious diseases. Several 61 approaches have been used for these studies including simple compartmental 62 models [31, 44], network models [35], and agent-based models [18, 24, 34, 48]. 
These models have provided new insights on important issues such as the effects of 63 drug resistance [5,46], treatment [34, 40], vaccination [3, 45], non-pharmaceutical 64 interventions $[11,15]$ and on the overall dynamics of infectious diseases [28]. 65

Diseases often spread through person-to-person contacts; therefore, realistic 66 mixing patterns can be crucial to accurately predicting the path and severity of 67 the disease [16]. The course of an epidemic through a population is determined by 68 the interactions among individuals and the process of transmitting a pathogen is a 69 stochastic (random) process based on the length of time the individuals are in contact 70 with each other and the strength of the contact. Agent-based models can capture this 71 realistic contact structure and allow the simulation to explore how contact networks 72 and different demographic characteristics affect disease transmission.

Several studies have shown the importance of population structure when 74 modeling disease spread [20], but only a few studies have incorporated 75 realistic mixing populations $[18,24]$. The accurate representation of population 76 heterogeneity is one of the greatest challenges of epidemic modeling. While 77 substantial progress has been made over the years with the introduction of different 78 mixing functions [29] and mixing matrices [2] for compartmental models, they are 79 still far from achieving a good approximation to real world scenarios. In recent 80 years, new approaches that incorporate more realistic contact structures have been 81 developed to allow for nonrandom interactions among populations [4, 22, 48, 54]. 82 For example, Zaric [54] compared random and nonrandom mixing patterns for 83 network epidemic models and showed that different mixing assumptions led 84 to different epidemic outcomes. In particular, they found that random mixing 85 generally results in a greater number of new infections than nonrandom mixing. 86 Similarly, Bansal et al. [4] used several real and simulated datasets of human 87 contact networks to analyze their impact on disease spread. They concluded that 88 homogeneous-mixing models are appropriate for host populations that are nearly 89 homogeneous. However, network models are more appropriate to better capture 90 and predict disease spread through heterogeneous host populations. Furthermore, 91 Fukś et al. [22] used an agent-based model of Southern and Central Ontario to 92 investigate the spatial correlations of disease spread. They concluded that spatial 93 correlations were difficult to destroy if neighborhood sizes were inhomogeneous. 94 Finally, Stroud et al. [48] showed a strong correlation between local demographic 95 characteristics and pandemic severity. This study used an agent-based model of 96 Southern California with a heterogeneously mixing population and concluded that 97 the average household size in a census tract was strongly correlated with the clinical 98 attack rate.

Here, we use a simple mathematical model to show how behavioral changes 100 can be easily introduced into epidemiological models. In addition, we use a large- 101 scale agent-based model to assess the potential impact of temporary and permanent 102 behavioral changes including school closures in containing a pandemic influenza 103 and analyze how these changes affect the contact structure and transmission 104 dynamics. 


\section{Methods}

We will consider two approaches to incorporate behavior changes in a mathematical 107 model. We first describe a simple system of five ordinary differential equa- 108 tions (ODEs) to describe disease dynamics based on the Kermack-McKendrick 109 susceptible-infected-recovered model (SIR) [31]. We extended this model by using 110 the approaches introduced in Del Valle et al. (2005) [15]. The second approach 111 is based on a stochastic agent-based model, object-oriented platform for people in 112 infectious epidemics (OPPIE). This is an extension of the Los Alamos Epidemic 113 Simulation System (EpiSimS) [16, 18,48] and includes dynamic behavior changes. 114

\subsection{Ordinary Differential Equation Model}

In our ODE model, the population is divided into two subgroups: a group that 116 does not change its behavior or has normal behavior (subscript $n$ ) and a group that 117 modifies its behavior in response to an outbreak (subscript $b$ ). People move back 118 and forth between the two groups (reducing susceptibility or infectivity) depending 119 on the behavior adopted. Individuals in each activity group are characterized by 120 their epidemiological status: susceptible, $S_{n}$ and $S_{b}$ and infectious individuals, $I_{n} 121$ and $I_{b}$; the transfers are shown diagrammatically in Fig. 4 . Because we are primarily 122 interested in the effectiveness of changes in behavior for a single outbreak, we use a 123 closed system with no migration in or out of the population, and births and natural 124 deaths are not included in the model.

We define $t_{0}$ as the beginning of the epidemic. Movement of individuals between 126 the two groups depends upon disease incidence in the population. It is assumed that 127 a certain fraction of the population will change their behavior to protect themselves 128 against infection or reduce their chances of spreading the disease. Let $\varphi_{S_{b}} S_{n}$ and 129 $\varphi_{I_{b}} I_{n}$ be the transfer rates from the $S_{n}$ and $I_{n}$ classes to the $S_{b}$ and $I_{b}$ classes, 130 respectively, and $\varphi_{S_{n}} S_{b}$ and $\varphi_{I_{n}} I_{b}$ be the transfer rates from the $S_{b}$, and $I_{b}$ classes 131 to the $S_{n}$ and $I_{n}$ classes, respectively. The rate coefficients are modeled by step 132 functions given by:

$$
\varphi_{i}=\left\{\begin{array}{l}
0, t<\tau \\
c_{i}, \tau<t<\tau_{\max } \\
0 t>\tau_{\max }
\end{array}\right.
$$

for $\mathrm{i}=S_{n}, I_{n}, S_{b}$, and $I_{b}$, where the parameter $c$ is a positive constant that determines 135 the rate of movement and $\tau$ is the time that determines when the new behavior is 136 adopted.

Using the transfer diagrams in Fig. 1, we obtain the following system of 138 differential equations: 


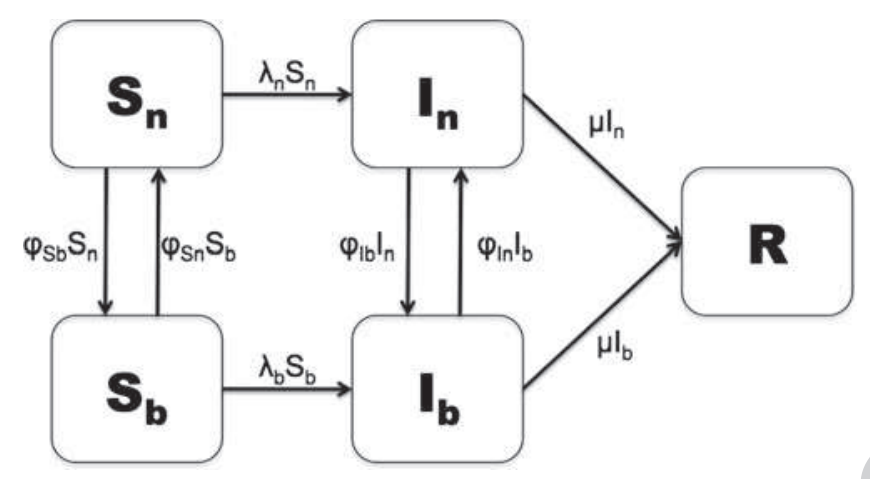

Fig. 1 Schematic relationship between people who adopt a new behavior in response to an epidemic and people who do not change their behavior. The arrows that connect the boxed groups represent movement of individuals from one group to an adjacent one. Susceptible individuals $\left(S_{n}\right.$ or $S_{b}$ ) can become infected $\left(I_{n}\right.$ or $\left.I_{b}\right)$ at rates $\lambda_{n}$ or $\lambda_{b}$; infected individuals recover at a rate $\mu$; and people change their behavior based on the transfer rates $\varphi_{S_{b}}, \varphi_{I_{b}}, \varphi_{S_{n}}$, or $\varphi_{I_{n}}$

$$
\begin{aligned}
\frac{d S_{n}}{d t} & =-\left(\varphi_{S_{b}}+\lambda_{n}\right) S_{n}+\varphi_{S_{n}} S_{b} \\
\frac{d I_{n}}{d t} & =-\left(\varphi_{I_{b}}+\mu\right) I_{n}+\varphi_{I_{n}} I_{b}+\lambda_{n} S_{n} \\
\frac{d S_{b}}{d t} & =-\left(\varphi_{S_{n}}+\lambda_{b}\right) S_{b}+\varphi_{S_{b}} S_{n} \\
\frac{d I_{b}}{d t} & =-\left(\varphi_{I_{n}}+\mu\right) I_{b}+\varphi_{I_{b}} I_{n}+\lambda S_{b} \\
\frac{d R}{d t} & =\mu\left(I_{n}+I_{b}\right)
\end{aligned}
$$

where $\lambda_{n}$ (for normal behavior) and $\lambda_{b}$ (for modified behavior) are the forces of 140 infection. $\lambda_{n}$ and $\lambda_{b}$ incorporate the probability of transmission per contact, $\beta, 141$ the reduced number of contacts because of symptomatic infection, $\theta$, and $1-\eta_{j} 142$ $(\mathrm{j}=s$ or $i$ ), which accounts for the effectiveness of the behavior in reducing 143 either susceptibility $\left(\eta_{s}\right)$ or infectivity $\left(\eta_{i}\right) . \beta$ is defined as the susceptibility of 144 the population multiplied by the infectivity of the disease multiplied by the average 145 number of contacts an individual has per day. The forces of infection for both groups 146 are shown by:

$$
\begin{aligned}
& \lambda_{n}=\beta\left[\left(\frac{\theta I_{n}}{\rho}\right)+\left(1-\eta_{i}\right)\left(\frac{\theta I_{b}}{\rho}\right)\right] \\
& \lambda_{b}=\beta\left[\left(1-\eta_{s}\right)\left(\frac{\theta I_{n}}{\rho}\right)+\left(1-\eta_{i}\right)\left(1-\eta_{s}\right)\left(\frac{\theta I_{b}}{\rho}\right)\right]
\end{aligned}
$$


where $\rho=N-(1-\theta)\left(I_{n}+I_{b}\right)$ and $N$ is the total population $\left(S_{n}+S_{b}+I_{n}+148\right.$ $\left.I_{b}+R\right)$. In the forces of infection, $\eta_{i}$ is incorporated into the $\theta I_{b} / \rho$ infectious 149 fractions because individuals in the $I_{b}$ class have adopted a new behavior and 150 $\eta_{s}$ is incorporated into the infectious fractions in $\lambda_{b}$ because individuals in the 151 susceptible class $\left(S_{b}\right)$ have also adopted a new behavior. These forces of infection 152 and appropriate initial conditions complete our model formulation.

\subsection{The Agent-Based Model}

The OPPIE simulation platform is an agent-based model that combines the 155 demographic-based population of a region, a network of specific business and home 156 locations, and the movement of individuals between locations with daily itineraries. 157 We simulated the spread of an influenza epidemic using a synthetic population 158 constructed to statistically match the 2000 US Census population demographics of 159 Southern California at the census tract level. There are 20 million individuals living 160 in six million households, with an additional one million locations representing 161 actual schools, businesses, shops, and social recreation addresses. This synthetic 162 population only represents individuals reported as household residents; thus, visiting 163 tourists, guests in hotels, and travelers in airports are not explicitly included. 164

Each individual has a schedule of activities based on the National Household 165 Transportation Survey (NHTS) [37]. A schedule specifies the type of activity, 166 the starting and ending time, and the location of each assigned activity. There 167 are six types of activities: home, work, shopping, social recreation, school, and 168 other. The time, duration, and location of activities determine which individuals 169 mix together at the same location at the same time, which is relevant for airborne 170 transmission.

Each location is geographically located using the Dun \& Bradstreet commercial 172 database. Each building is subdivided based on the number of activities available at 173 that location. There are one or more buildings per activity that are further subdivided 174 into rooms or mixing places. Schools have classrooms, workplaces have workrooms 175 or offices, and shopping malls have shops. Typical room sizes can be specified; 176 for example, for workplaces the mean workgroup size varies by standard industry 177 classification (SIC) code. The number of rooms in each building is computed by 178 dividing the peak occupancy by the appropriate mixing group size. We used two data 179 sources to estimate the mean workgroup by SIC including a study on employment 180 density [53] and a study on commercial building usage from the Department 181 of Energy [36]. Based on these two data sources workgroup sizes range from 182 3.1 for transportation workers to 25.4 for health service workers. The average 183 workgroup size over all types of work is 15.3 . For the analyses presented here, 184 the average mixing group sizes are 8.5 at a school, 4.4 at a shop, and 3.5 at a social 185 recreation venue. 


\subsubsection{Disease Progression Model}

Airborne diseases spread primarily from person to person during close proximity 188 through contact, sneezing, coughing, or via fomites. In OPPIE, an opportunity for 189 disease spread between two individuals occurs when they occupy a mixing location 190 together. Whether or not a susceptible individual becomes infected is based on how 191 long they co-occupy within a mixing place, the presence of infectious individuals, a 192 high-level description of the activity they are engaged in, and their age. 193

A location represents a street address, and a room or mixing place represents 194 a specific place where people have face-to-face interactions. When an infectious 195 person is in one of these mixing locations with a susceptible person for some time, 196 we estimate a probability of disease transmission, which depends on the variables 197 identified above.

A susceptible person $j$ has a dimensionless susceptibility multiplier $S_{j}$ and an 199 infectious person $i$ has an infectious multiplier $I_{i}$. The probability that the susceptible 200 individual $j$ becomes infected during an activity is computed as:

$$
P_{j}=1-e^{-\sum_{i} T S_{j} I_{i} t_{i j}}
$$

where $T$ is the average transmissibility per unit time, $t_{i j}$ is the duration of contact, 202 and the sum extends over all infectious people that occupied the room with 203 individual $j$.

Disease progression of the epidemic is modeled as a Markov chain consisting 205 of five main epidemiological stages: uninfected, latent (non-infectious), incubation 206 (partially infectious), infectious, and recovered. Infected individuals start in the 207 incubation stage and remain there for a period of between 0 and 0.5 days, 0.5 or 208 1.0 day, before transitioning to the symptomatic or recovered stages, respectively. 209 The average incubation time is 1.9 days and average duration of the symptomatic 210 stage is 4.1 [34]. The disease model assumes that $50 \%$ of adults and seniors, 211 $75 \%$ of students, and $80 \%$ of preschoolers will stay home within 12 hours of 212 the onset of influenza symptoms. These people can then transmit disease only to 213 household members or visitors. Based on previous studies [34], 33.3\% of infections 214 are assumed to be subclinical. Individuals in the subclinical stage are only half as 215 infectious as those in the symptomatic stages and continue their normal activities 216 as if they were not infected. The infection rate for children is assumed to be 217 double than for adults. All scenarios were analyzed for the same set of transmission 218 parameters where the population was initially seeded with 100 people infected, all 219 in the incubation stage.

The Homeland Security Council released the National Strategy for Pandemic 222 Influenza for the United States, which suggests that the emergence of a new 223 influenza virus could have a clinical disease attack rate of $30 \%$ in the overall 224 
population [49]. Based on this attack rate, we constructed a baseline scenario under 225 the assumption of no specific intervention to contain the pandemic and an infection 226 attack rate of $45 \%$ with a clinical attack rate of $30 \%$.

\subsubsection{School Closure Assumptions}

Protecting children during an influenza pandemic is important since illness rates are 229 typically highest among school-aged children [38]. Closing schools limits students' 230 contacts and has the potential to block paths of spread between families and 231 neighborhoods [1]. Several studies have analyzed the impact of school closures 232 $[8,21,24]$; however, these studies only investigated the impact of sustaining a school 233 closed during the entire epidemic. School closures in OPPIE are implemented as 234 a general closure of selected activity locations. Based on the Center for Disease 235 Control and Prevention pandemic guidelines [9], closures in OPPIE follow a steplike 236 function and are specified with a start and stop time; the activity to close; and a single 237 location or a fraction of all locations of the specified activity type that will be closed. 238 During the time a closure is in effect, anyone whose activity schedule would have 239 taken them to one of the closed locations will stay home during that time instead. 240 Scheduled after-school activities are not affected by a school closure.

\subsubsection{Fear-Based Home Isolation Assumptions}

Fear-based home isolation consists of people staying home as a reaction to an 243 epidemic crisis. Some of these people may be considered the "worried well". 244 The news of increasing numbers of people becoming ill, or seeing friends and family 245 fall ill, is strong motivation to avoid potential infectious situations. Surprisingly, 246 none of the recent studies on pandemic influenza have incorporated the impact 247 of this type of behavioral change. We assume that people isolate due to fear at 248 a level that follows the pattern of the epidemic [6,27]. This is implemented with 249 a specification of start, peak, and end times with corresponding fractions of the 250 population that will be isolating at those times, along with a minimum and maximum 251 contiguous duration per individual. We assume that people who choose to stay home 252 will only self-isolate for 7-14 days at a time. People isolate on an individual basis, 253 not on a household basis, so there might be households in which some members 254 of the family are isolating due to fear and others are going about their normal 255 activities. Fear-based home isolation begins when a percentage of the population 256 is symptomatic (e.g., 0.1\%). The number of people self-isolating increases linearly 257 until reaching a maximum near the epidemic peak day. After this day, the stay-home 258 rate begins to drop linearly with time, until no fear-based home isolation is occurring 259 by a selected end day. 